\title{
El elefante en la habitación: dualidad y costes de despido en Espańa
}

\author{
The elephant in the room: duality and severance costs \\ in Spain
}

Juan J. Dolado*

Departamento de Economía. Universidad Carlos III de Madrid

ORCID ID: 0000-0003-3790-2457

doi: https://doi.org/10.20318/labos.2021.6222

\section{Introducción}

Durante el pasado mes de Noviembre de 2020 ví el episodio “Tiempos Modernos" de la nueva temporada de Salvados (la Sexta). En el mismo, el nuevo presentador del programa (Gonzo) recogía por la mańana a la Ministra de Trabajo, Yolanda Díaz, de la puerta del Ministerio y la trasladaba en coche a varias localidades de sur de Madrid donde abunda precariedad y paro. La ministra, muy empática en todo momento, se enfrentó a las quejas y miserias de jóvenes y no tan jóvenes hasta bien entrada la noche cuando el presentador la devolvió a Nuevos Ministerios. Como era previsible para una mujer inteligente y bien curtida en lides sindicales, Díaz reconoció los graves problemas que asolan a nuestro mercado laboral y aportó algunas soluciones en marcha que podrían ser prometedoras.

Me sorprendió, sin embargo, que en ningun momento abordó cual era el origen del problema de nuestra dualidad. Es el elefante en la habitación que todos los políticos ignoran. Me refiero a la clase política y no a todo el resto de la sociedad porque muchos investigadores sobre este tema han discutido hasta la saciedad sobre el cáncer de nuestro modelo dual (véase un buen resumen de estas opiniones el Capitulo 7 del informe España 2050, 2021). Un páis donde machaconamente se firman un $90 \%$ (o más) de contratos temporales (CT) al año, las tasas de de conversión de CT a contratos indefinidos (CI) no suben del 10\%, se reduce drásticamente la duración de los CT (en promedio duran 49 días) y la tasa de temporalidad casi nunca de baja del $25 \%$, no puede seguir así, con pandemia o sin ella.

El hecho de que este tema nunca se aborde seriamente en la larga lista de reformas laborales que llevamos en nuestro país obviamente implica que alguien sale beneficiado del statu quo existente. Probablemente, los empresarios piensen que la diferencia en la proteccion al empleo (días de salario por ańo trabajado) entre CI y CT sea demasiado alta, incluso tras la reforma de 2012 (de 45/24 dias vs 8 dias a 33/20 dias vs. 12 días). Detrás de ello estas los llamados costes red tape por el hecho (asociado a incertidumbre sobre decisiones judiciales) de que los CI puedan ser apelados frente a la magistratura laboral mientras que la finalización de un CT ( pero no su rescisión, sujeta a

*dolado@eco.uc3m.es 
las mismas normas que los CI) no puede ser objeto de apelación. Otra posibilidad es que la cultura de la temporalidad lleve instalada en nuestra clase empresarial per secula seculorum: reduce sus costes laborales y el resto de efectos nocivos (alto desempleo, baja productividad y formacion, escasa fertilidad y gran precariedad) no parece afectarles. Debe ser que, al carecer de experiencia empresarial, desconozco alguna razón mas profunda que justifique sus prácticas habituales .

Por su parte, los sindicatos saben que su supervivencia depende de los intereses de los trabajadores con CI, que son los que se afilian (aunque cada vez menos) y votan en las elecciones sindicales, y por tanto actúan en consecuencia. Todo ello pese a sus declaraciones públicas en contra de la precariedad, donde parece que el "problema" no va con ellos. Tampoco con el Ministerio que parece seguir apostando por el control del fraude contractual con una plantilla de cerca de 2100 Inspectores y Subinspectores de Trabajo para monitorizar cerca de 20 millones de contratos en un año de actividad económica normal. De ilusión también se vive.

¿Debemos rendirnos y aceptar la situación actual? Personalmente creo que hay que seguir intentándolo, por supuesto desde nuestra investigación, que desde luego no es sagrada pero si mas seria que las opiniones ideológicas que frecuentemente se escuchan en los medios.

\section{Contrato Unificado}

En un artículo recientemente publicado (véase Dolado, Lalé y Siassi, 2021) nos planteamos analizar cual sería el tránsito en el mercado laboral en España desde un modelo de contratación con discontinuidad en las indemnizaciones por despido tras un período predeterminado ( 2 años en nuestro caso), como el vigente en la actualidad, a otro sin discontinuidad, mediante lo que se ha denominado un contrato unificado. Las principales cuestiones que intentamos abordar se centran en simular cúáles son serían sus efectos sobre las variables claves del mercado laboral en estado estacionario y, sobre todo, durante la fase de transición de un sistema de regulacion de la protección al empleo a otro alternativo.

En nuestro modelo hay dos tipos de trabajadores: jóvenes/adultos (denominados "Y", con edades entre 25-55) y mayores (“O”: 55-64). En consonancia con la amplia evidencia existente para la contratación temporal España, tras dos años de antigüedad en el mercado laboral, los trabajores entrantes se enfrentan a costes de despido mas elevados (el modelo está calibrado a la economía española de 2005-07, previa a los efectos de la Gran Recesión y a la reforma de 2012 cuando la tasa de paro era del 9\% y el gap típico en indemnizaciones era de 45-8=33 dias). La elección de este período corresponde a una situación económica que pensamos se ajusta a lo que debería ser en el equilibrio estacionario en tiempos normales: parecernos a Europa. Los trabajores son aversos al riesgo y pueden ahorrar para suavizar el consumo durante aquellos períodos en que se encuentren parados. Además, tienen derecho a recibir indemnizaciones y prestaciones por desempleo sufragadas con cotizaciones, que tambiém les ayudan a transitar hacia la jubiliacion en el caso de los trabajadores mayores, cuyas tasas de reempleo son menores por sus mayores salarios de reserva y el avance del progreso tecnológico. Todos los trabajadores también acumulan capital humano en el puesto de trabajo a través de la formación, tienen diferente productividad de entrada, y negocian bilateralmente (a la Nash) sus salarios (parcialmente rígidos por la existencia del SMI y suelos salariales pactados en convenio) con las empresas.

El modelo calibrado en sus parámetros para reproducir las principales magnitudes (stocks y flujos) del mercado de trabajo pre-Gran Recesión (Tabla 1), se ajusta muy bien a los datos, proporcionando cifras muy similares a las observadas en las tasas de paro y no-empleo (inactividad) para ambos tipos de trabajadores. Una vez calibrado el modelo, el paso siguiente es simular los efectos 
contrafactuales de la introducción del contrato unificado de forma no retroactiva, esto es mantiendo la anterior regulación para aquellos períodos de empleo previos a la implementacón de la reforma y las reglas de la nueva posteriormente. Dichos efectos se muestran en la Figura 1. Como puede observarse, antes de introducir la reforma, la tasa de destrucción de empleo se dispara al 10\% a los 2 años de un contrato pese, aunque el aumento se ya se produce bastante antes, dado que la empresan descuentan la subida en indemnizaciones a pagar a aquellos trabajadores despedidos después de 2 años. Además reducen la formación específica de los trabajadores ante sus escasa estabilidad laboral. Por su parte, estos últimos anticipan que sus empleos estan en peligro por la brecha en las indemnizaciones, por lo que reducen sus salarios durante los 2 primeros años para compensar a las empresas por el aumento de sus costes de depido. Sin embargo, no pueden hacerlo en la cuantía necesaria debido a la existencia de rigideces salariales a la baja.

\begin{tabular}{|lcc|}
\hline Non-employment rates & Data & Model \\
\hline unemployment, young workers & 9.0 & 9.7 \\
non-employment, older workers & 43.1 & 36.3 \\
non-employment, all workers & 16.5 & 16.4 \\
\hline Distribution of job tenure & Data & Model \\
\hline less than 2 quarters & 8.7 & 6.8 \\
2 to 4 quarters & 8.8 & 5.7 \\
1 to 3 years & 15.9 & 15.9 \\
3 to 5 years & 13.2 & 10.9 \\
5 to 10 years & 16.5 & 18.8 \\
more than 10 years & 36.9 & 41.9 \\
\hline
\end{tabular}

Notes: The table provides a comparison between model-generated moments and their empirical counterparts computed from the Spanish Labour Force survey for the years 2005-2007. All entries are expressed in percent.

Tabla 1. Variables laborales en estado estacionario (modelo y datos 2005-2007).

(a) Probability at job tenure $\tau$

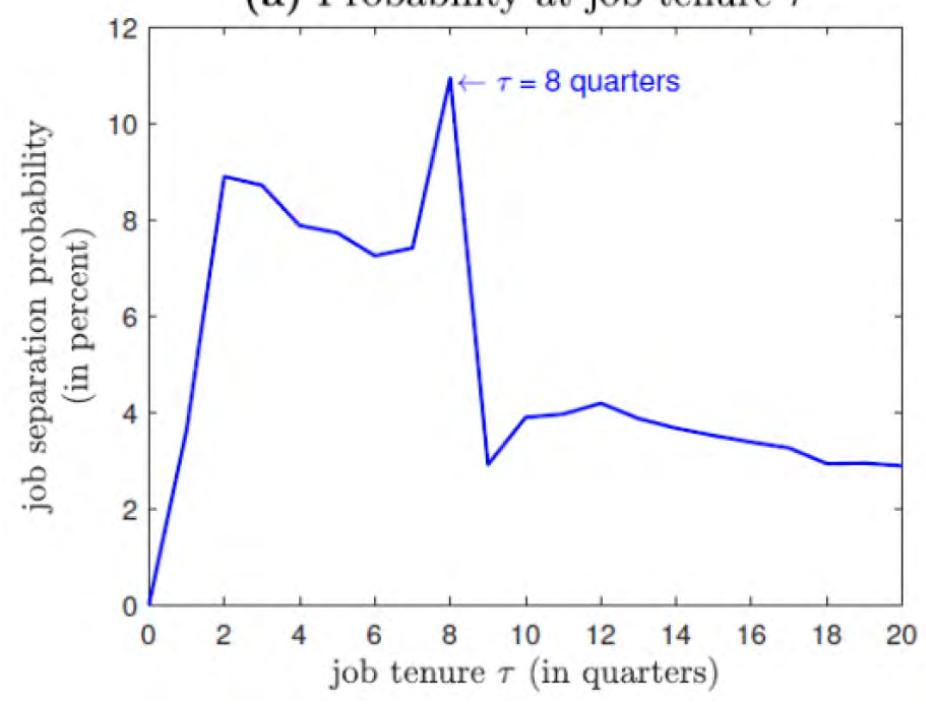

Figura 1. Tasa de destrucción de empleos. 
El siguiente paso es obtener el perfil de un contato unificado que maximice el flujo de bienestar laboral a lo largo de sus carreras profesionales de los nuevos trabajadores que acceden al mercado de trabajo. Con los parámetros adoptados para reproducir los hechos estizados de las magnitudes laborales antes de la Gran Recesión, encontramos que el contrato unificado óptimo tiene un período de prueba de 5 meses (sin indemización) con una indemnización posterior de 20 días. En la Figura 2 se muestra el perfil de las indemizaciones en 2005-2007 (línea continua con, recordemos 8 dias antes de 2 años y 45 dias postriormente) y con el nuevo contrato (linea rayada).

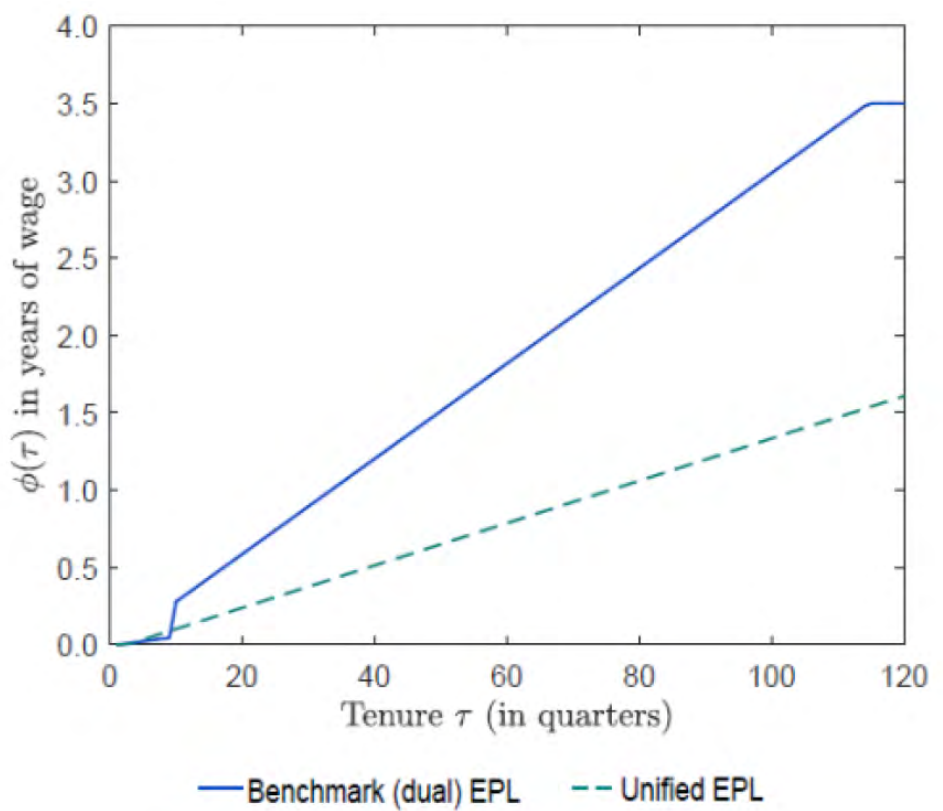

Figura 2. Perfil de indemnizaciones con la antigüedad.

Además, en dicho artículo se demuestra que todas las variables relevantes experimentan un cambio favorable durante la fase de transición que conduce al contrato unificado. Por lo que respecta al nivel de bienestrar (medido en unidades de consumo equivalente), éste aumenta en un 1.93\% para los trabajadores tipo "Y", al tiempo que se reduce en un $0.79 \%$ para los trabajadores tipo "O", especialmente entre aquellos con salarios mas bajos cuyo despido es más barato. No obstante, la ganancia neta de bienestar es positiva pues la proporción en la población activa de trabajadores tipo "Y" es mucho más alta que la del tipo "O". La Tabla 2 presenta las ganancias de bienestar y los restantes efectos sobre las variables laborales en el estado estacionario.

En resumen, la aprobación de esta reforma en el sistema de costes de despido conlleva la existencia de ganadores y perdedores, pero las ganancias de bienestar de los primeros son más que suficientes para compensar las pérdidas de los segundos. La razón de este resultado favorable es que las empresas se muestran menos reacias a extender los contratos más allá de 2 años, a la vez que crean más puestos de trabajo y mejoran la formación de sus trabajadores. Es cierto que también destruyen más empleos al reducirse los costes de despido, pero el efecto creación es más fuerte que el efecto destrucción, de manera que aquellos trabajadores que pierden su empleo rápidamente consiguen otro y aumenta la protección por desempleo al incrementarse la recaudación de las cotizaciones sociales con una mayor y más estable tasa de empleabilidad. Todo ello está en línea con el modelo de "flexiseguridad" imperante en los países nórdicos y en parte de los anglosajones.

El modelo también racionaliza la existencia de protección al empleo que aumente con la antigüedad en el puesto de trabajo, ya que los trabajadores "O" con antigüedad larga en el puesto 


\begin{tabular}{|lccc|}
\hline Aggregate equilibrium variables & Dual EPL & Unified EPL & Change (\%) \\
\hline tightness & 1.00 & 1.27 & 27.5 \\
payroll tax & 9.03 & 7.41 & -18.0 \\
\hline Non-employment and job turnover & Dual EPL & Unified EPL & Change (\%) \\
\hline unemployment, young workers & 9.52 & 7.51 & -21.2 \\
non-employment, older workers & 33.1 & 31.3 & -5.5 \\
job-finding rate & 40.0 & 50.1 & 25.3 \\
job destruction, $\leq 2$ years & 7.44 & 6.36 & -14.5 \\
job destruction, >2 years & 2.22 & 2.38 & 7.3 \\
\hline Unemp. duration (young workers) & Dual EPL & Unified EPL & Change (\%) \\
\hline less than 6 months & 40.0 & 50.0 & 25.0 \\
6 months to 1 year & 36.8 & 36.8 & 0.1 \\
1 year to 2 years & 18.7 & 11.9 & -36.1 \\
more than 2 years & 4.48 & 1.16 & -74.1 \\
\hline Welfare in consumption equivalents & Dual EPL & Unified EPL & Change (\%) \\
\hline new entrants & - & - & 1.93 \\
\hline
\end{tabular}

Tabla 2. Efectos de introducir un contrato unificado.

de trabajo se enfrentan a menores oportunidades de reempleo en caso de despedido. Investigamos el papel de este mecanismo cambiando la duración esperada de la fase de vejez (durante la cual los trabajadores no reciben ofertas de trabajo). Si una política de bonus malus consiguiera reducir los despidos de los trabajadores mayores y acortar de 10 a 5 años el período en que no buscan empleo, entonces la pendiente del contrato unificado óptimo con la antigüedad se reduciría de 20 a 12 días de indemnización por año trabajado, mientras que, si fracasara y dicho período aumentara a 15 ańos, dicha pendiente subiría a 32 días, sin cambios significativos en el período de elegibilidad que no acarrea indemnización.

Nótese que, para que un modelo sea computacionalmente manejable, se requiere hacer abstracción de algunas cuestiones que pueden ser relevantes en contextos concretos. Por ejemplo, las ventajas comparativas de la economía española en determinados sectores intensivos en CT (la Florida del sur de Europa con fuerte implantación de actividades en construcción, hostelería y el turismo) implica la presencia ineludible de este tipo de contratación en dichos sectores, por lo que el contrato unificado debería coexistir con CT restringidos a aspectos formativos y de sustitución.

\section{Mochila austriaca}

Junto a este tipo de propuesta de reforma, muchos expertos (incluyendo el Banco de España en su informe anual de 2020) abogan por la introducción de la denominada mochila austriaca que recibe su nombre de la reforma de la legislación de protección al empleo que tuvo lugar en Austria en 2003 (Kramarz et al., 2021). Todos los trabajos a partir del 1 de enero de dicho año pasaron a estar sujetos a un nuevo sistema de pensiones ocupacionales. A partir del segundo mes de la relación laboral, el empresario debía transferir el 1,53 por ciento del salario actual del trabajador a una cuenta de pensiones que devenga intereses, propiedad de este último. Cuando se agota la relación laboral, ya sea por despido (no disciplinario) o renuncia, dicha cuenta permanece en manos del empleado que puede retirar los fondos tras 3 años de tenencia para su propio uso, bien acumulando más capital humano o complementado su pensión a la hora de la jubilación. El objetivo de esta reforma era fomentar la movilidad laboral al desligar una parte de la indemnización de la antigüedad en la misma empresa, al tiempo que permitía suavizar a lo largo del tiempo las aportaciones de las empresas al fondo de indemnizaciones, en vez de concentrarlas en las fases recesivas del ciclo económico cuando les es más complicado acometer estas obligaciones. El estudio del Banco de España (2021) sobre 
este tema encuentra que una aportación de las empresas de 6 días por año trabajado a los fondos individuales de sus trabajadores y una reducción del $50 \%$ de los costes de despido y finalización de CT (pasando a 16,5 días por despido improcedente, 10 por despido objetivo y 6 por fin de CT) resultarían ser neutrales respecto a las cuantías recibidas por los asalariados y pagadas por las empresas bajo la legislación vigente. Los costes de la transición de un sistema al otro supondrían unos 9 mil millones de euros que, ante el déficit actual de la Seguridad Social, se aconseja sean financiados con parte de los 140 mil millones de fondos europeos de Nueva Generación (NGEU) que va a recibir España en los próximos seis años.

Tanto la propuesta de modificación de los costes de despido tras la reforma de 2012 como la introducción de la mochila austriaca llevan mucho tiempo encima de la mesa del gobierno y los agentes sociales, sin ningún avance sustancial. Como se ha comentado previamente, la patronal prefiere mantener la rotación continua de los CT como la única vía conocida de reducción de costes laborales en caso de ajuste de plantilla. El grave problema de baja productividad asociada a la inestabilidad de este tipo de contratos no parece importarles demasiado, especialmente en sectores con barreras a la competencia impuestas por las empresas de mayor tamaño en la negociación sectorial. Pese a sus proclamas contra la precariedad, los sindicatos prefieren seguir centrándose en proteger a los asalariados con CI que les aseguran su reelección en un sistema de negociación colectiva con cláusula erga omnes (inédita en los países de la UE), baja representatividad de ambas partes (especialmente empresarial) y escasa afiliación por parte de los trabajadores. Por tanto, en presencia de una resistencia numantina a dichos cambios regulatorios, cortar el nudo gordiano del problema fundamental del mercado laboral en Espańa requiere de otra clase de medidas políticamente factibles que, a la vista de los retos presentes y futuros que afronta nuestro mercado de trabajo, habrán de ser audaces, tipo big bang, en vez de tener carácter gradual (Dolado, Felgueroso y Jimeno, 2021).

\section{Nueva clasificación de contratos laborales}

En estas circunstancias, una propuesta factible desde el punto de vista de la economía política sería incorporar los contratos eventuales y de obra y servicio (un 56\% de los CT en 2020 Q3: 2.22m) a la categoría de CI (16.1m. en la actualidad). En 2018, hubo 262 mil despedidos con CI y 150 mil finalizaron sus CT. Los primeros recibieron una indemnización media de 17 mil euros mientras que los segundos percibieron 1.130 euros. Con estas cifras, la indemnización media por despido en dicho año fue de 11.200 euros (= 17000x 262/412+1350x150/412). Si el 56\% de los CT pasaran a ser indefinidos (y sin variar la compensación de 12 días por a la finalización de los restantes CT), el coste medio por despido de los trabajadores con CI que mantiene constante la cifra anterior de 11,200 euros de indemnización media pasaría a ser de unos 15.770 euros, frente a los 17 mil anteriores. Para calcular la nueva indemnización se ha supuesto que los contratos de obra y servicio y eventuales tienen una duración un tercio inferior a los de los CI ordinarios. Por tanto, la indemnización por despido objetivo habría de reducirse de 20 a unos 18.5 días $(=20$ x $15,77 / 17)$ de salario por ańo trabajado si todos los despidos fueran de esta naturaleza. Si, por el contrario, solamente el $50 \%$ de los despidos fueran objetivos, entonces la reducción habría de ser mayor. En efecto, manteniendo los 33 días por despido improcedente, en este caso la indemnización por despido objetivo que resulta neutral en costes es de 14 días, cifra que aumentaría a 16 días si se redujera la proporción de despidos improcedentes al 25\% (delimitando más claramente las causas del despido objetivo).

Esta estrategia de abordar el perenne problema de la dualidad en nuestro mercado de trabajo parece estar en línea con la reciente propuesta del Ministerio de Trabajo a los agentes sociales sobre posibles cambios a introducir a la fallida reforma de 2012 en el tema de la excesiva e ineficiente dua- 
lidad. Sería muy útil para eliminar de una vez por todas la elevada tasa de temporalidad de nuestra economía que con esta medida pasaría del $24.2 \%$ al $10.4 \%$. Sin embargo, se olvida que para que sea políticamente aceptable no puede implicar una subida del actual nivel de costes de despido que sigue a la cabeza de los países de nuestro entorno (p.ej., para un trabajador con 10 años de antigüedad, la indemnización media es de 28,6 semanas frente a 21,7 en Alemania, 14 en Portugal, 8,7 en Francia o 7 semanas en Reino Unido). La realización de cómputos más exactos que los llevados a cabo anteriormente parece ineludible para que la propuesta siga adelante y no tenga que ser impuesta por la Comisión Europea como requisito a la percepción de los fondos NGEU. Solo de esta manera la presencia del elefante se haría perceptible en la habitación.

\section{Referencias}

BANCO DE ESPAÑA (2021). Informe Anual 2020.

DOLADO, J., FELGUEROSO, F. and J. F. JiMENO (2021). "Past, Present and Future of the Spanish Labour Market: When the Pandemic Meets Megatrends" Applied Economic Analysis, 29 (85), 21-41.

DOLADO, J., LALÉ, E. and N. SIASSI (2021). From Dual to Unified Employment Protection: Transition and Steady State" Quantitative Economics, 12 (2), 547-585.

KRAMARZ, F., MALHEBERT, F., TURON, H., KETTEMANN, A. and J. ZWEIMÜLLER (2021). "Job Mobility and Creative Destruction: Flexicurity in the Land of Schumpeter", mimeo. 